\title{
Live Broadcasting of TV-Stream Over 5G in Commercial Applications
}

\begin{abstract}
Thobokgolo Masabase
Student, Dept. of Computing and Technology, Anglia Ruskin University, Cambridge and Chelmsford, United Kingdom

Abstract: The development of mobile devices and their intensifying use has been overwhelming in the last decade. This has led to evolution of complex mobile networks to cope with the tremendous need for data consumption. Mobile applications and services have become complex and sophisticated to meet end-users expectations such as multimedia communication. 4G mobile communication system is the latest and current technology offering higher data transmission rates than its predecessor $3 \mathrm{G}$. Currently $4 \mathrm{G}$ can reach up to $1 \mathrm{Gbps}$. With challenge from ever growing need for data, research has been kicked off for $5 \mathrm{G}$ which is expected to offer increased maximum throughput, highly secure system and low battery consumption. This paper proposes an application for multimedia streaming on cameras that are fully mobile to capture video and stream it over 5G networks. This application utilizes $5 \mathrm{G}$ technology to deliver improved end-user and commercial experience.
\end{abstract}

Keywords: 5G, Multimedia, Flat IP, Wireless Communication, Quality of Service

\section{INTRODUCTION}

The world of future wireless technology has gone through remarkable transformation on the domain of communication. This change is significantly noticeable by how everyone is embracing the use of technology on universities, workplaces and in hotspots. From $1 \mathrm{G}$ to $3 \mathrm{G}$, telecommunication improved rapidly to offer better enduser experience than before. Currently $4 \mathrm{G}$ and LTE are being rolled out and this is changing the lives of many individuals, encouraging advanced application revolutions. With the current $4 \mathrm{G}$ mobile communications, it has been noticed that the data bandwidths can be improved which kicked research on 5G or LTE-Advanced technologies.

5G technologies promise to offer higher bandwidths $1 \mathrm{Gbps}$ and above, which are highly needed for applications like VOIP, multimedia streaming and with support for dynamic information access. Multimedia streaming is defined as one or more media type transmission which can be text, graphics, voice, video and audio [16]. This concept has brought the birth of IPTV systems which revolutionized the entertainment and media industries. This kick started development of better delivery of content from internet sources which is linked to quality of service (QoS) [2].

With requirement to satisfying users, robust technologies are emerging. IPv6 is the latest replacing the IPv4 which is running out of address space. IPv6 with more address spaces enables features to be added to it like QoS joined with operative routing procedures [2]. As a result of this, flat IP is drawn from IPv6 as an important feature that is aimed at developing 5G. Flat IP ensures real-time delivery of on-demand services to customers by removing the old hierarchical architecture [15]. Because address space is now vast, this allows for every node to be addressed and located accurately. This helps in development of cognitive radio which is the cornerstone of $5 \mathrm{G}$ with consideration to mobility factor.
Cognitive radio simply means radio which is wireless transmission of signals will have knowledge of free channels and which channel to occupy, type of data transmitted and environment awareness [14]. With mobility involved, cognitive radios must continue to work and adapt to the changing environment to ensure continues transmission of data [1], [3]. Access techniques FDMA, TDMA, CDMA and OFDM are utilized to divide spectrum efficiency within a limited frequency space [14]. Using network technologies in place; LAN /WLAN, fully functional network design enables nodes/access points to cooperate within their proximities. This is sometimes referred to as machine to machine communications and smart grids utilize this kind of setup to wirelessly transmit data. Based on these network technologies and techniques, developing applications that consume high data rates becomes easy and end user experience is improved to maintain high degree of quality of service.

\section{5G TECHNOLOGY CONCEPTS}

\section{A. $5 G$ Technology}

$5 \mathrm{G}$ technology is not currently available on shelve, this is a name used to describe technology research beyond 4G. $5 \mathrm{G}$ will be designed to offer high bandwidth of $1 \mathrm{Gbps}$ and higher. Other features will be to solve the spectrum licensing and aiming at lower battery consumption [7]. Table 1 below shows the comparison of features from the $3 \mathrm{G}$ to the proposed 5G. Bandwidth improvements and change in services provided is noticeable through this evolution.

To achieve the above desired behavior, terminals are to be designed with software-defined radios, integrated with different modulation schemes and error-control schemes which will solve frequency licensing and improve spectrum utilization and management [7], [10]. 
Table 1: Comparison of features in 3G, 4G and 5G [10]

\begin{tabular}{|c|c|c|c|}
\hline $\begin{array}{l}\text { Technology } \\
\text { /Features }\end{array}$ & $3 G$ & $4 G$ & $5 G$ \\
\hline Bandwidth & $2 \mathrm{Mbps}$ & $\begin{array}{l}2 \mathrm{Mbps} \\
1 \mathrm{Gbps}\end{array}$ & $\begin{array}{l}\text { 1Gbps and } \\
\text { higher }\end{array}$ \\
\hline $\begin{array}{l}\text { Technology } \\
\text { used }\end{array}$ & $\begin{array}{l}\text { Broad } \\
\text { bandwidth, } \\
\text { CDMA, IP }\end{array}$ & $\begin{array}{l}\text { Unified IP, } \\
\text { LAN, WAN, } \\
\text { WLAN }\end{array}$ & $\begin{array}{l}\text { Unified IP, } \\
\text { LAN, WAN, } \\
\text { WLAN plus } \\
\text { WWWW }\end{array}$ \\
\hline $\begin{array}{l}\text { Multiple } \\
\text { Access }\end{array}$ & CDMA & CDMA & $\begin{array}{l}\text { CDMA, } \\
\text { OFDMA } \\
\text { and BDMA }\end{array}$ \\
\hline Core network & $\begin{array}{l}\text { Packet } \\
\text { network }\end{array}$ & Internet & Internet \\
\hline $\begin{array}{l}\text { Service } \\
\text { provision }\end{array}$ & $\begin{array}{l}\text { Integrated } \\
\text { high quality } \\
\text { audio, } \\
\text { video and } \\
\text { data }\end{array}$ & $\begin{array}{l}\text { Dynamic } \\
\text { information } \\
\text { access, } \\
\text { wearable } \\
\text { devices e.g. } \\
\text { Google } \\
\text { Glass }\end{array}$ & $\begin{array}{l}\text { Dynamic } \\
\text { information } \\
\text { access, } \\
\text { wearable } \\
\text { devices with } \\
\text { Artificial } \\
\text { Intelligence } \\
\text { (AI) }\end{array}$ \\
\hline
\end{tabular}

This application incorporates artificial intelligence on each terminal eventual forming a network with more awareness. This provides a support for high bandwidth at low cost for heavy bandwidth application such as interactive multimedia, voice and streaming video [11], [16].

Figure 1 shows a typical network design for 5G mobile systems. The system is made of user terminals, independent, autonomous radios access nodes which are treated as IP link to the Internet [15]. IPv6 is used to give each node an address to ensure proper routing of packets.

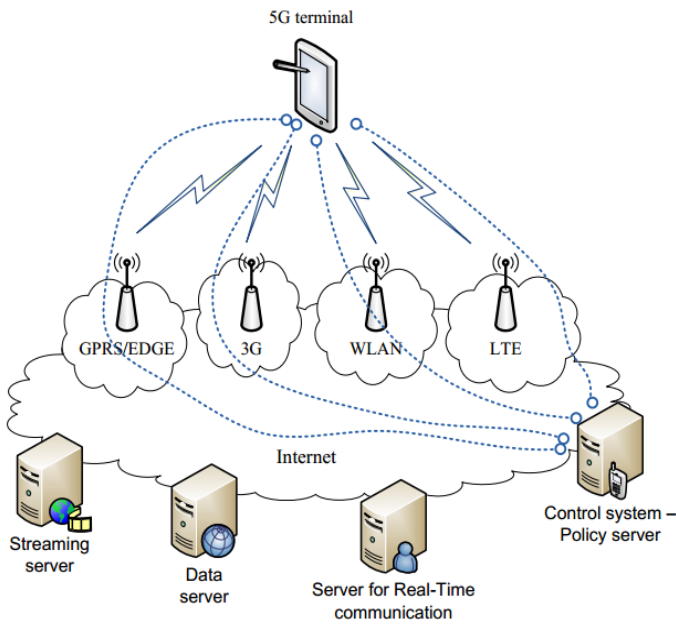

Figure 1: Design of 5G Network [15]

To make the system work to provide its competitive advantage, 5G incorporates various technologies and techniques which already exist and new ones and major ones are discussed below respectively.

\section{B. $\quad$ World Wide Wireless Web}

Artificial Intelligence integrated into radio within $5 \mathrm{G}$ technologies will be integral for increased services provision. This technology allows interconnection of
devices to enable multi-bandwidth data paths for $5 \mathrm{G}$ wireless world hence termed World Wide Wireless Web (WWWW) [3]. WWWW will be capable of delivering full wireless-based Web application support for real-time multimedia with speeds beyond 1Gbps [6], [13]. With this technology concept, it is possible to add required number nodes to the network to enhance or increase throughput and incorporate intelligence as software based solutions. This kind of designs is user-centric as they target at meeting user expectations.

\section{BDMA as Multiple Access Technique for $5 G$}

Since research for $5 \mathrm{G}$ has been kicked, a Korean research and development has suggested that BDMA (Beam Division Multiple Access) technique can be used as radio interface for $5 \mathrm{G}$, which is not dependent on frequency/time resources [14]. This is one way of solving spectrum management and licensing costs compared to existent multiple access techniques.

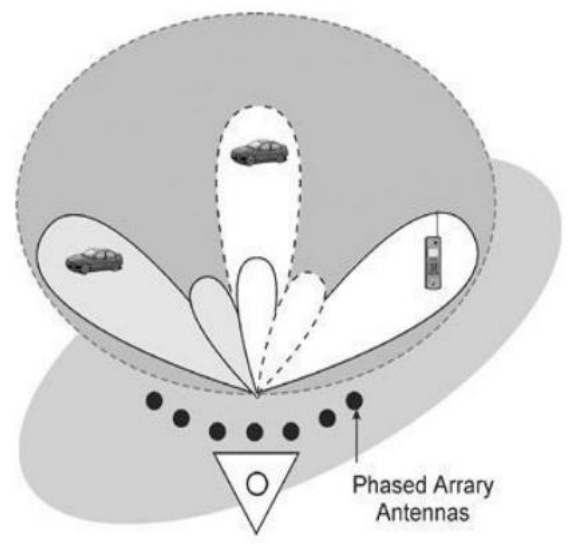

Figure 2: Beam Division Multiple Access [14]

A base station in BDMA network allocates separate beam to each mobile station so that it divides the antenna beam according to the location of the mobile stations [14] and [15]. With multiple access capability to the antenna by mobile stations, capacity is increased significantly as both the base and mobile station are in line of sight with each other. Line-of-sight in wireless communication implies direct communication without interference. The antenna forms multiple beams to mobile stations using phase array antenna to target them at different angles. This technique adds to the existing CDMA, OFDM which are dependent on time and frequency.

\section{Addressing using IPv6}

Due to mobility involved in $5 \mathrm{G}$ systems, each must be node uniquely identifiable no matter its position. To address this problem, IPv6 is used in addressing by providing unique addresses. IPv6 addresses are 128bit, which is four times the $32 \mathrm{bit} \mathrm{IPv} 4$ which provide enough address spaces [14]. As a unified world network, hierarchy is removed and Flat IP is introduced. Flat IP is being used by many wireless operators to delivery improved services to customers. With IPv6, improved control features can be added and data streams can be serviced with different level of service hence increasing Quality of Service (QoS). 


\section{E. Flat IP Architecture}

This architecture uses symbolic names to identify devices hence eliminating hierarchical architecture such as the use of "normal" IP addresses [15]. This is critical for 5G technologies to lower costs, provide a seamless universal access and improve end user experience [11]. Radio access and packet core networks are decoupled hence evolving independently of each other to create flexibility in network planning and deployments. Scalability in Flat IP is efficient as fewer nodes are used removing those excess nodes which were used to form hierarchical architecture resulting in reduction of operational costs for wireless operators and increasing quality of service.

\section{F. Cognitive Radio}

From the introduction section, Cognitive Radios are aware of free channels and bandwidths availability enable them occupy channels not in use, know the type of data transmitted. Knowledge enhancement into radio is cardinal for channel sensing, recognizing modulation scheme in use and position of other nodes [14]. This knowledge is designed in software to enable reconfiguration of parameters when needed by user demands. This allows for flexibility with growing demands of mobile data as spectrum use is optimized, resources are allocated and consumed evenly [9], [10].

\section{5G RESEARCH IN PROGRESS}

$5 \mathrm{G}$ research is at a starting phase investigating on robust routing mechanisms to improve data transmission rates. Progress is being made on varies technologies like cognitive radio, invention of wearable devices and recently Google Glass has been introduced. Korean researchers have proposed a multiple access technique Beam Division Multiple Access (BDMA) which they suggested for use in 5G [14]. In the United Kingdom, University of Surrey has started research on $5 \mathrm{G}$ by securing up to $£ 35 \mathrm{M}$ funding from the government and a consortium of mobile operators and telecommunication infrastructure providers [17]. This shows that there is progress and investment into researching better ways of improving broadband mobile internet services.

5G technologies involve many players from users, service providers, companies like Google, Cisco and researchers to standardization bodies like IEEE and 3GPP. Users are at the core as service is based on meeting their demand expectations. With every person's data consumption increasing and varying, mobile data services consumption puts pressure on service providers to deliver better data rates. Service providers will experience need for increased in bandwidths hence searching for effective ways of delivering improved services to users while keeping their operating costs low.

Cisco Corporation has being involved in forecasting trends in mobile data traffic for the whole world and their results show increase in data consumption per device [5]. Results $A$. show that the amount of data used by smart devices (tablets, smart phones) will increase showing a positive correlation to the type of application mostly used like video and M2M. Handheld devices which can be easily used while moving will consume more data by 2017 , hence a need for $5 \mathrm{G}$ implementation [5]

Standardization bodies like IEEE, 3GPP, ITU has not yet started on standardizing this technology as they regard everything after $3 \mathrm{G}$ and $4 \mathrm{G}$ to be Long Term Evolution research. Recently, spectrum frequency used for TV at $700 \mathrm{MHz}$ frequencies has been auctioned for research and for use in $4 \mathrm{G}$ technologies. WWRF publishes papers on solutions for wireless and how to make them interoperable in a heterogeneous environment [4].

\section{PROPOSED APPLICATION DESIGN}

The proposed application involves delivery of multimedia streaming which is television video to users over the $5 \mathrm{G}$ communication networks in real-time. The design architecture of the proposed application consists of the media source where the video cameras are located together with intermediate Access Points forming Radio Access Network (RAN) [15]. This network is then connected to the core network where the TV broadcaster station is located for distribution of multimedia content to viewers. From the core network, multimedia is distributed to end users through different channels, which can be internet streaming, cable for video on demand or satellite [16]. This allows delivering TV stream service to any user using any device at any location, whether static or mobile. Figure 3 below shows the 5G system architecture for the proposed application.

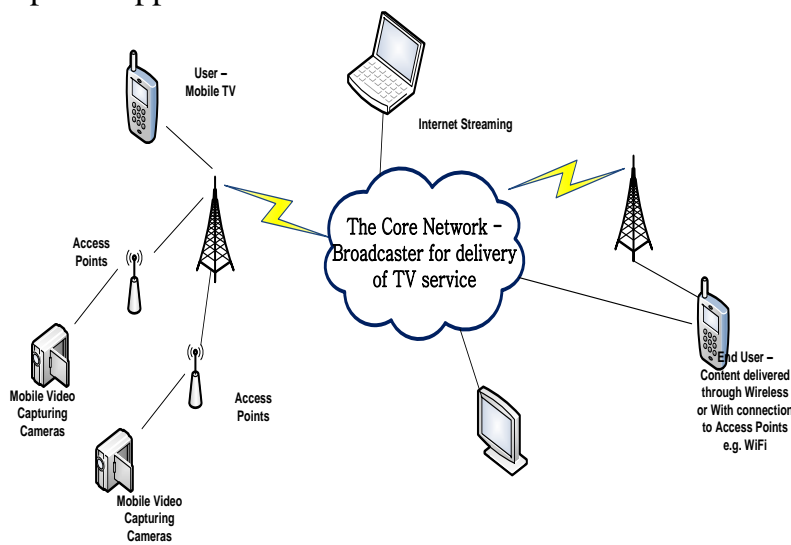

Figure 3: Proposed Application Design

On this system, video cameras which are fully mobile capture video recordings for television broadcasting and immediately transmit it to the broadcasting station wirelessly for distribution in real-time. This application can be used for recording live events such as sports where there is mobility, and in movie shooting industry. Considering mobility and real-time delivery factors, this application can perform exceptionally on $5 \mathrm{G}$ due to availability of increased bandwidth needed between the broadcaster and the video source and between the broadcaster and the end users.

A.

Mixed Bandwidth Data Path

The video camera in this case acts like a mobile node which will subsequently communicate with access points www.ijarcce.com 
in the nearby surrounding to the core network. The path 5G technology concepts. All devices should support IP data follows on this network can be mixed i.e. and should support IPv6 so that they can be part of the transmission over different media paths with different core network [14]. The advantages of IPv6 enabled transmission rates and capabilities to increase bandwidth devices will impact positively on the delivery of content to for multimedia streaming. The access points and the base users as parameters such as quality of service can be stations support radio access and radio link management capabilities for each mobile node [3].

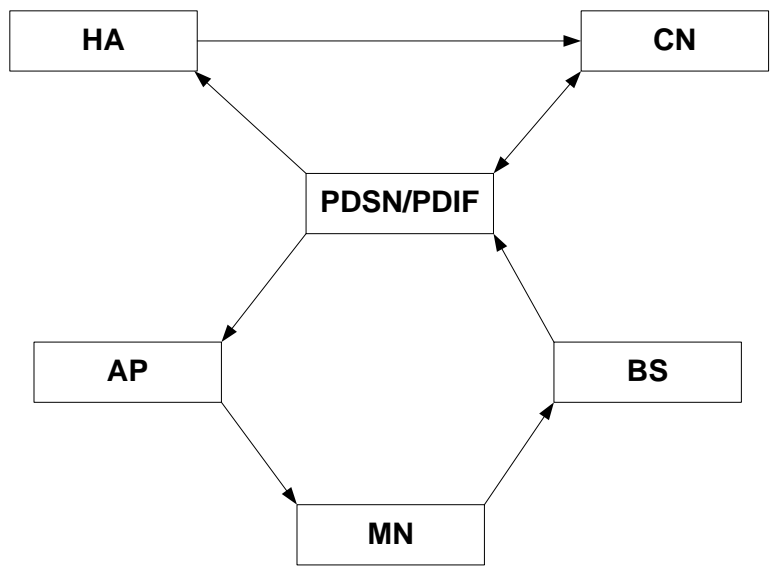

Figure 4: Mixed Bandwidth Data Path Model [3], [12]

From this model, the Mobile Node (MN) can either send a request or transmit through the Base Station (BS) via the Packet Data Service Network (PDSN) to the Core Network $(\mathrm{CN})$ [12]. The reply path will be through the Access Point (AP) to the MN from the CN. This model forms mixed bandwidths because the MN can support PDSN from BS and Packet Data Internetworking Function (PDIF) from the AP [12]. If both PDSN and PDIF are exposed to the $\mathrm{MN}$, then the two networks provide increased bandwidth to the mobile node simultaneously.

Exposing this type of network to the proposed application will provide maximum bandwidth needed for video streaming compare to transmission using microwaves on old television systems. The APs and BSs in this architecture form the RAN with ability for topology discovery, bootstrapping and auto-configuration [13]. This enables APs and BSs to be monitored and maintained closely as they can provide system logs used for debugging and troubleshooting. Compared to the satellite and aerial systems, the system provides more opportunity for research and improvement on design architecture by using the raw information from this system. This architecture utilize the cognitive radio concept by having $\mathrm{AP}$ and BS being environment aware and being able to sense unoccupied channels. The ability to recognize the type of data being transmitted adds an advantage to the network as resources are optimized for use in transmission. This touches on artificial intelligence and cognitive radio concepts which enables devices to be fully away of their environment and adapt to changing conditions like topology or changing traffic patterns.

\section{B. Device Support}

To better utilize this proposed application, devices used within the system should at least support quite a number of controlled. Evolution of devices currently is incorporating IP into devices such as TV, game consoles even connecting home control systems. With such devices in homes, this provides a base for $5 \mathrm{G}$ technology which provides coverage with smaller cell sites like femtos and pico cells and upgraded backhaul to ensure we can get the most out of the whole system [8].

\section{Multimedia and its Protocol Support}

It should be understood that increased bandwidth on its own does not bring improved end user experience; multimedia on its own should be of high quality. The quality of multimedia like video includes its high definition, clear audio without any loss of information. On this application, high multimedia quality will be maintained by using high quality capturing cameras. This will ensure that media delivered to users is of high quality from the source. The protocols used in transporting the multimedia should maintain and protect high quality of multimedia [16]. Real-time transfer protocol (RTP) is used for transporting real-time media such as speech, video streams and audio [16]. When using protocols such as RTP on this application, they should follow flexible standardization that will allow for more easy codecs and presentation control irrespective of device platforms. For example, videos on mobile phones should be presented with same quality as they would appear on HDTV. Interoperability is essential in making sure that any device should be able to present this kind of media to users especially for mobile devices which are vendor specific. With IPv6, these media types must be recognizable to allow for improved quality of service on transmission and network nodes should be able to sense and recognize them too.

\section{Efficiency and Benefits}

The aim of $5 \mathrm{G}$ technology is to deliver support for higher bandwidths to users and with the ability to support large broadcasting of data to almost 65,000 connections [4]. This shows high speed data transmission for large data rates. With TV streaming supporting this technology, we can expect to have High Definition Television (HDTV) which will be clear, with added capability for multiple channel delivery. End users will not only get improved user experience but also cost for these services are expected to be low. With 5G, devices can connect together and share broadband internet, hence eliminating need for a central access point. Power consumption is also expected to be low for devices using $5 \mathrm{G}$ technology. Performance of devices like the video cameras for this proposed application is expected to be improved while consuming less power. For maximum performance and continued use, devices are expected to be built with mechanism to optimize their use in low power situations. This includes hibernation mechanisms to save high power utilization and 
also to notify other neighbouring nodes of their current power status so that bandwidth and transmission paths can be engineered to adapt to limited available resources. This will be a matter of optimization between available battery life on nodes and the volume of what is been transmitted on the network.

\section{E. Challenges and Limitations}

The current research is to find ways of dealing with the present challenges facing technologies like 4G. To set up networks like this one for $5 \mathrm{G}$ need a great effort considering a number of factors like security, maintenance and operation management. Achieving data rates higher than $1 \mathrm{Gbps}$ with minimal power consumption is a challenge as higher data rates equates to more processing effort hence more power consumption. Research has been done on finding the best batteries that can be used for devices and ways of storing more power on smaller batteries.

\section{F. Availability of Similar Application}

Currently, applications similar to the proposed one are not available on the market. Applications like smarts grids use similar concept but for different application use like on electricity bills reading. Even though the end user setup currently exists, it is not fully $4 \mathrm{G}$ as $3 \mathrm{G}$ and GSM are still being used in some situations.

\section{FUTURE DEVELOPMENT}

With smart devices being developed with increased functionality and capability, this technology is likely to exist in the future. This is supported by improvements made on devices like HDTVs with Wi-Fi integrated, improved input/output terminals like HDMI for high data rates support. Devices like portable cameras which are capable of capturing media that is ready to publish e.g. on YouTube and are enabled with wireless connectivity capabilities shows the high likelihood of this application being developed. Machine to machine (M2M) communication is the much talked about concept and with its current deployment on smart grids technology (e.g. on utility meters which communicate wirelessly) shows that this technology application is likely to be developed.

\section{CONCLUSION}

With the ongoing research on the mobile technologies to overcome challenges and limitations of $4 \mathrm{G}$ mobile communication, breakthrough will be made and better data rates will be introduced. New enhanced protocols like IPv6 and efficient standard like streaming and codecs for streaming multimedia are being developed thus adding progress towards $5 \mathrm{G}$ technology. From this research, the current existing technologies and those that are proposed for 5G offers a solid stand for finalizing 5G implementation. New technologies like BDMA and cognitive radio will play an important role in the implementation of $5 \mathrm{G}$ as they aim at eliminating challenges with the limited spectrum usage and licensing costs. The proposed application is most likely to be implemented once $5 \mathrm{G}$ is available by looking at the current technologies which use similar concept. Device evolution and high quality multimedia presentation formats also suggests the likelihood of such application with increase in smart devices, TV sets becoming IP and Wi-Fi enabled with improved screen resolutions.

\section{ACKNOWLEDGEMENT}

I would like to thank my friend Percy Mokabedi for providing me with access to IEEE articles during my paper research at Carleton University library.

\section{REFERENCES}

[1] Abbas, A., Khan, K., \& Khan, J. (2011, May). Cellular Handover Approaches in $2.5 \mathrm{G}$ to $5 \mathrm{G}$ Technology. International Journal of Computer Applications, Volume 21(No.2).

[2] Al-madi, A. M., Idrus, R., Ramadass, S., \& Budiarto, R. (2008, March). A Proposed Model for Policy-Based Routing Rules in the IPv6 Offering QoS for IPTV Broadcasting. International Journal of Computer Science and Network Security, Vol.8(No.3).

[3] Aryaputra, A., \& Bhuvaneshwari, N. (2011, October 19-21). 5G The Future of Mobile Network. Proceedings of the World Congress on Engineering and Computer Science, Vol. II.

[4] Camiciotti, L., Lamy, C., \& S.Verdi, P. (2004, May). Joint sourcechannel coding for $4 \mathrm{G}$ multimedia streaming. Journal Computer Communications, Vol.27(No 08), 762-779.

[5] CISCO Corporation, C. (2013, Febrruary 06). Cisco Visual Networking Index: Global Mobile Data Traffic Forecast Update. Retrieved June 08, 2013, from www.cisco.com: http://www.cisco.com/en/US/solutions/collateral/ns341/ns525/ns53 7/ns705/ns827/white_paper_c11-520862.html

[6] Cunningham, G., Murphy, J., Murphy, L., \& Perry, P. (2009, December). Seamless Handover of IPTV Streams in a Wireless LAN Network. Broadcasting - IEEE Transactions, Vol. 55(No. 04), $796-801$.

[7] Dumbre, N., Patwa, K., \& Patwa, M. (2013, February). 5G WIRELESS TECHNOLOGIES-Still $4 \mathrm{G}$ auctions not over, but time to start talking 5G. International Journal of Science, Engineering and Technology Research, Volume 2(Issue 2).

[8] Frenzel, L. (2011). Wireless Companies Follow the Roadmap Past 4G And On To 5G. Electronic Design, Vol. 59(No. 08), pp. 28-34.

[9] Janevski, T. (2009). 5G Mobile Phone Concept. 6th IEEE Consumer Communications and Networking Conference, Vol. 1013 , pp. pages $1-2$.

[10] Janevski, T., \& Tudzarov, A. (2011, April). Protocols and Algorithms for the Next Generation 5G Mobile Systems. Vol.3(No. 1).

[11] Kristiansson, J., \& Parnes, P. (2004, March 21-25). Applicationlayer Mobility Support for Streaming Real-Time Media. IEEE Wireless Communications and Networking Conference, Vol 1, 268273.

[12] Li, X., Salleh, R., \& and Zakaria, O. (2009). Multi-network Data Path for 5G Mobile Multimedia. 2009 International Conference on Communication Software and Networks (pp. 583 - 587). IEEE Computer Society.

[13] Mohd, M. S. (2011, July 19-20). Visions of 5G Communications. International Conference, HPAGC 2011, pp 252-256.

[14] Patel, S., Chauhan, M., \& Kapadiya, K. (2012, September). 5G: Future Mobile Technology-Vision 2020. International Journal of Computer Applications, Volume 54(No.17).

[15] Patil, S., \& Patil, V. (2012, January). A Review on 5G Technology. International Journal of Engineering and Innovative Technology, Volume 1(Issue 1).

[16] Tanwar, G., \& Singh, G. (2010). Multimedia Streaming Technology in 4G Mobile Communication Systems. International Journal on Computer Science and Engineering, Vol.02(Issue 03), 695-699.

[17] University of Surrey Press, R. (2012, October 8). THE UNIVERSITY OF SURREY SECURES £35M FOR NEW 5G 
International Journal of Advanced Research in Computer and Communication Engineering Vol. 3, Issue 11, November 2014

RESEARCH CENTRE. Retrieved May 17, 2013, from www.surrey.ac.uk:

http://www.surrey.ac.uk/mediacentre/press/2012/90791_the_univer sity_of_surrey_secures_35m_for_new_5g_research_centre.htm

\section{BIOGRAPHY}

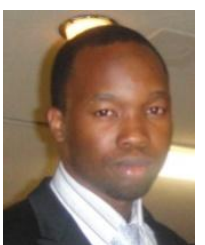

Mr Thobokgolo O. Masabase, received Bachelor's Degree

Telecommunications with a Minor in Business from Carleton University in Canada, 2012. He is currently pursuing MSc in Information Technology from Anglia Ruskin University in United Kingdom. He has worked as a software developer at CaseWare IDEA Inc, Canada. He is interested on mobile communications, big data, modern technology applications and data mining. 